\title{
Éditorial
}

\section{Les systèmes de soins de longue durée dans cinq pays*}

Comme la population mondiale vieillit, la santé des personnes âgées et les soins à leur prodiguer sont des enjeux sociaux et de recherche. En 1988, en réaction à cette situation, un projet tout à fait particulier et stimulant voit le jour: ICE on Aging - le programme de collaboration internationale sur le vieillissement. Il est parrainé par le National Center for Health Statistics désormais intégré aux centres de prévention et de contrôle des maladies, au sein du département de la santé et des services aux personnes des ÉtatsUnis.

L'objectif du projet ICE on Aging comporte trois volets: (a) effectuer une recherche collective à caractère international qui favorise notre compréhension de la santé des aînés; (b) améliorer l'évaluation et la comparabilité internationale des données sur la santé des populations vieillissantes; (c) développer et soutenir des réseaux internationaux de chercheurs dont les travaux portent particulièrement sur la santé des aînés.

ICE on Aging est lancé en 1988, au cours d'un colloque international (National Center for Health Statistics, 1991); des participants proposent alors une recherche comparative sur la santé et le vieillissement. Les discussions permettent de mettre en lumière six domaines où la recherche s'avère particulièrement essentielle: (a) les statistiques sur les affections cardiovasculaires et le cancer, (b) la promotion de la santé et la prévention des maladies, (c) l'incapacité fonctionnelle, (d) les maladies chroniques courantes, (e) la vitalité, (f) les soins de longue durée. À la suite de ce colloque, six chercheurs venant de l'Australie, du Canada, des Pays-Bas, de la Norvège et des ÉtatsUnis unissent leurs efforts et mettent en commun leurs bases de données pour entreprendre une recherche sur l'un de ces domaines, celui des soins de longue durée (SLD) dans un monde vieillissant. Les conditions de base de ce projet collectif remontent à 1985 , l'année la plus proche pour laquelle il est possible d'obtenir des données comparables dans ces cinq pays.

Il existe beaucoup de similitudes entre les populations âgées des cinq pays. La proportion de personnes âgées (65 ans et plus) en Norvège s'élève à 16 pour cent; dans les quatre autres pays, elle se situe entre 11 pour cent et 13 pour cent. L'espérance de vie à la naissance est de 78 à 80 ans chez les femmes, et 72 à 74 ans chez les hommes. À l'âge de 65 ans, la variation de l'espérance de vie dans ces pays est d'au plus un an, soit: 18,5 ans chez les femmes, et 14,5 ans chez les hommes. Au sein du groupe des 85 ans et plus - la cohorte qui risque de présenter le plus grand besoin de soins de longue durée - l'espérance de vie atteint 5,7 ans chez les femmes, et 4,8 ans chez les hommes. Dans les cinq pays, ce dernier groupe représente 8 pour cent à 10 pour cent de la population âgée de 65 ans et plus, et ce pourcentage 
connaît une hausse rapide (Van Nostrand, Clark, \& Romøren, 1993).

Les établissements de soins de longue durée des cinq pays visés constituent un deuxième point de comparaison. Si le projet est principalement axé sur les soins de longue durée, les chercheurs doivent, de toute évidence, établir des normes d'équivalence pour définir ce que chacun et chacune désignent centres d'hébergement. Par conséquent, il a été décidé que l'analyse porterait principalement sur les niveaux les plus élevés de soins institutionnels fournis dans chaque pays, c'est-à-dire un minimum de soins infirmiers, l'assistance en soins personnels ainsi que le gite et le couvert (Van Nostrand et al., 1993).

Mais les analyses des SLD publiées dans le présent numéro de la revue ${ }^{1}$ dépasse sensiblement ces similitudes fondamentales et s'étendent à des domaines aussi divers que la prestation des services et la qualité des soins, la gérontopsychiatrie, les finances et les principes pour la construction de banques de données. Au sein même de chacun des pays, la situation offre des perspectives multiples et une diversité notable. Par exemple, aux ÉtatsUnis, l'accent mis sur les soins institutionnels, comme l'analysent Van Nostrand (1996, présent numéro), est pondéré par l'importance croissante et le potentiel d'une autre solution qui n'a pas encore été pleinement mise en oeuvre dans aucun des cinq pays, soit les soins à domicile et les soins communautaires dispensés aux aînés (Clark, 1996, présent numéro).

Il est aussi intéressant de souligner que dans tous ces pays les systèmes de soins de santé ont subi des changements d'une grande portée depuis 1985, année à laquelle renvoient les données de référence recueillies aux fins de ce projet. L'équilibre est au centre des deux plus grandes préoccupations en matière de santé relativement à l'élaboration d'une politique de soins de longue durée. D'abord, il faut établir un équilibre entre les soins institutionnels et les soins à domicile, compte tenu du vieillissement de la population et de la modification des structures familiales. Ensuite, il faut parvenir à un équilibre entre la centralisation et la décentralisation des services. Au dire de Romøren (1996, présent numéro), le coût de la décentralisation affaiblit les politiques nationales et accentue les différences géographiques dans chacun des pays (p. 71).

Havens et Bray (1996, présent numéro) font état des mêmes défis et contraintes fiscales qui sont devenus le moteur de la politique de réforme de la santé à travers le Canada. En conséquence, le groupement administratif et l'intégration des programmes ont été substitués par la décentralisation des services et par la définition de régions de taille réduite. La croissance anticipée des services communautaires a été exceptionnellement modeste, mais les compressions de tous genres qui ont touché les soins institutionnels ont été plus draconiennes que prévu.

Dans le contexte de l'évolution du système de soins en Australie et aux Pays-Bas, Howe (1996, présent numéro) et van den Heuvel (1996, présent numéro) révèlent l'existence de politiques semblables qui restreignent l'expansion des centres d'hébergement et autres établissements de soins de longue durée tout en développant le secteur des soins communautaires dans 
les deux pays. En Australie, le rôle des foyers pour personnes âgées a été redéfini de façon radicale pour faire face aux changements survenus dans les soins institutionnels et dans les soins communautaires. Les Pays-Bas, pour leur part, ont misé davantage sur les soins communautaires comme solution de rechange. Van Nostrand (1996, présent numéro), se reportant à la politique américaine sur les soins de longue durée, relève une tendance marquée vers les soins institutionnels. Toutefois, en se reportant au travail complémentaire de Clark (1996, présent numéro), elle laisse également supposer qu'un important revirement en prestation de soins de longue durée se dessine comme le démontre une récente croissance dans les services de soins de santé à domicile et de soins communautaires.

Puis, van den Heuvel (1996, présent numéro) nous rappelle qu'en dernière analyse, la solution repose sur la philosophie culturelle du pays et sur l'attitude de la population à l'égard de ses aînés (p. 57). Parallèlement, Romøren (1996, présent numéro) fait remarquer qu'à longue échéance, la légitimité du système est liée à la perception qu'en ont les bénéficiaires ... et en dernier ressort, au fait que la majorité de l'électorat partage la même opinion (p. 71).

Clark (1996, présent numéro) souligne un autre point important en matière de politique et de planification, en nous rappelant que les bases de données sur les soins de longue durée se sont sensiblement développées au cours de la dernière décennie. Toutefois, comme le mentionnent la majorité des auteurs, il manque encore des données longitudinales utiles à l'examen des causes et effets et à la description des transitions vécues par les personnes âgées dans les domaines de la santé, de la capacité fonctionnelle, de la longévité, de l'utilisation des services ou de la source de financement. Ces données sont essentielles pour faire face au changement, pour prévoir les besoins et pour connaitre les résultats des politiques.

Ce point est soulevé dans pratiquement tous les articles du présent numéro. Par exemple, Howe remarque qu'au cours des dernières années en Australie, les données tant quantitatives que qualitatives ont servi à évaluer les soins fournis. Elle laisse supposer que l'amélioration de la base de données constitue l'une des plus grandes réalisations du pays en matière de soins aux personnes âgées. Les systèmes informatiques actuels permettent aux Australiens d'examiner, d'analyser, de raffiner et d'adapter les politiques et les programmes. Elle ajoute - et nous partageons tout à fait cet avis - que le projet ICE on Aging nous offre la possibilité unique d'échanger des informations et de profiter des expériences des autres pays. En outre, grâce à la comparabilité des données qui a été étudiée, la recherche comparative peut être approfondie.

D'autres recherches comparatives sont en cours. Elles serviront de fondement aux exposés que présenteront les auteurs, à l'automne 1996, dans le cadre du colloque International Collaborative Effort on Aging qui regroupe des spécialistes du monde entier. Ce numéro spécial du Canadian Journal on Aging/La Revue canadienne du vieillissement offre aux collaborateurs spécialisés en soins de longue durée de ICE on Aging l'occasion de 
partager ces données et les résultats des évaluations avec un très grand nombre de collègues. Il est à souhaiter que ces efforts favorisent l'élargissement de la base de la recherche comparative pour l'étendre à d'autres pays, au moment où les soins de santé - y compris les soins de longue durée - continuent d'évoluer dans le temps et sur toute la planète. L'Année internationale des personnes âgées que les Nations Unies ont décrétée en 1999 pourrait bien fournir la prochaine impulsion à l'expansion de la recherche, tout comme l'accroissement rapide du nombre des plus âgés des aînés à l'aube du 21e siècle stimule l'élaboration de politiques et de programmes en divers soins de longue durée.

\section{Notes}

* Les auteurs désirent remercier le National Center for Health Statistics du soutien continu dont il a assuré le groupe de travail sur les soins de longue durée de ICE on Aging, et du financement qu'il lui a fourni. Nous tenons également à remercier de leurs commentaires pertinents, le rédacteur en chef et les quatre réviseurs anonymes du $C J A / R C V$, qui ont lu les versions antérieures des articles de ce numéro. En dernier lieu, nous disons un merci tout particulier à Nina L. Colwill qui a héroïquement accompli la tâche titanesque de créer - à partir de nos textes rédigés en des styles bien différents et comportant tout près de 300 pages dactylographiées - un numéro spécial que nous croyons cohérent et agréable à lire. Toute erreur ou omission sont entièrement de notre responsabilité.

1 Les personnes désirant obtenir de plus amples données sur les questions relatives aux soins de longue durée traitées dans l'un ou l'autre des articles du présent numéro, doivent communiquer avec chacun ou chacune des auteurs qui leur transmettront des tableaux détaillés.

\section{Références}

Clark, R.F. (1996). Home and community-based care: The U.S. example. La Revue canadienne du vieillissement, 15(hors série 1), 91-102.

Havens, B., \& Bray, D. (1996). International comparisons of long-term care: Cana$\mathrm{da}$, with specific reference to Manitoba. La Revue canadienne du vieillissement, 15(hors série 1), 31-45.

Howe, A.L. (1996). International comparisons of long-term care: Australia. La Revue canadienne du vieillissement, 15(hors série 1), 13-30.

National Center for Health Statistics. (1991). Proceedings of the 1988 international symposium on data on aging. Vital Health Statistics, 5(6), 1-265.

Romøren, T.I. (1996). International comparisons of long-time care: Norway and the Scandinavian solution. La Revue canadienne du vieillissement, 15(hors série 1), 59-72,

van den Heuvel, W.J.A. (1996). Long-term nursing home care in The Netherlands. La Revue canadienne du vieillissement, 15(hors série 1), 46-58

Van Nostrand, J.F., Clark, R.F., \& Romøren, T.I. (1993, juin). Nursing home care in five nations. Ageing International, 20(2), 1-5.

Van Nostrand, J.F. (1996). The focus of long-term care in the United States: Nursing home care, La Revue canadienne du vieillissement, 15(hors série 1), 7390. 